\title{
A horse of a different colour: Do patients with semantic dementia recognise different versions of the same object as the same?
}

\author{
M. Ikeda ${ }^{a, *}$, K. Patterson $^{b}$, K.S. Graham ${ }^{\text {b }}$, M.A. Lambon Ralph ${ }^{\text {c }}$, J.R. Hodges ${ }^{b, d}$ \\ a Department of Neuropsychiatry, Ehime University School of Medicine, Shitsukawa, Toon City, Ehime 791-0295, Japan \\ ${ }^{\mathrm{b}}$ MRC Cognition and Brain Sciences Unit, Cambridge, UK \\ c Department of Psychology, University of Manchester, UK \\ d Neurology Unit, Addenbrooke's Hospital, Cambridge, UK
}

Received 12 November 2004; received in revised form 29 June 2005; accepted 1 July 2005

Available online 22 August 2005

\begin{abstract}
Ten patients with semantic dementia resulting from bilateral anterior temporal lobe atrophy, and 10 matched controls, were tested on an object recognition task in which they were invited to choose (from a four-item array) the picture representing "the same thing" as an object picture that they had just inspected and attempted to name. The target in the response array was never physically identical to the studied picture but differed from it - in the various conditions - in size, angle of view, colour or exemplar (e.g. a different breed of dog). In one test block for each patient, the response array was presented immediately after the studied picture was removed; in another block, a 2 min filled delay was inserted between study and test. The patients performed relatively well when the studied object and target response differed only in the size of the picture on the page, but were significantly impaired as a group in the other three type-of-change conditions, even with no delay between study and test. The five patients whose structural brain imaging revealed major right-temporal atrophy were more impaired overall, and also more affected by the 2 min delay, than the five patients with an asymmetric pattern characterised by predominant left-sided atrophy. These results are interpreted in terms of a hypothesis that successful classification of an object token as an object type is not a pre-semantic ability but rather results from interaction of perceptual and conceptual processing.
\end{abstract}

(C) 2005 Elsevier Ltd. All rights reserved.

Keywords: Temporal lobe atrophy; Semantic knowledge; Object recognition; Visual perception; Generalisation

\section{Introduction}

The topic of this study is object recognition, operationally defined as the normal human ability to classify two perceptually different tokens of an object type as 'the same thing'. The evidence to be presented comes from patients with semantic dementia (SD), arising from progressive circumscribed atrophy in anterior, inferior regions of temporal neocortex (Hodges, Patterson, Oxbury, \& Funnell, 1992; Imura, 1943; Snowden, Goulding, \& Neary, 1989; Tanabe et al., 1992). This disorder of semantic memory disrupts knowledge of objects as well as words, as measured by tasks such as matching pictures on an associative or functional basis and copying

\footnotetext{
* Corresponding author. Tel.: +81 89960 5315; fax: +81 899605317.

E-mail address: mikeda@m.ehime-u.ac.jp (M. Ikeda).
}

line drawings of familiar objects after a brief delay (Bozeat, Lambon Ralph, Graham, Patterson, Wilkin, Rowlwnd, Rogers, \& Hodges, 2003; Bozeat, Lambon Ralph, Patterson, Garrard, \& Hodges, 2000; Lambon Ralph \& Howard, 2000; Lauro-Grotto, Piccini, \& Shallice, 1997; Rogers et al., 2004a; Snowden \& Neary, 2002). Relatively little is known, however, about the impact of such semantic deterioration on object recognition as defined in the first sentence of this paragraph.

One approach to this issue has adopted, as its gold standard, the task of object decision, in which participants are asked to discriminate between pictures of real and non-real objects, the non-real ones typically being incorrect combinations of real object parts. Many researchers have assumed that accurate performance in this task requires only activation of appropriate representations in a structural description system that precedes access to, and is independent of the status 
of, conceptual knowledge about the objects (e.g. Humphreys, Riddoch, \& Quinlan, 1988). If this assumption were correct, then one might expect patients with SD to perform normally in object decision, because they generally succeed on higher level tests of visual perception that do not require semantic knowledge. This prediction might be further bolstered on neuroanatomical grounds. Lesion studies, although rarely offering precise localisation, have associated the posterior right quadrant of the brain with abilities like object decision and unusual-views matching (e.g. Warrington \& James, 1988). Recent functional imaging studies of object decision by normal participants specifically implicate very posterior temporal or even occipito-temporal cortex, particularly in the right hemisphere (e.g. Gerlach, Law, Gade, \& Paulson, 1999; Kellenbach, Brett, \& Patterson, 2001; Kellenbach, Hovius, \& Patterson, 2005). Since there is typically no significant structural abnormality in these posterior regions in SD (Galton et al., 2001; Mummery et al., 2000), the twin presumptions: (a) that object recognition based on structural descriptions is functionally localised here and (b) that this function is quite separate from semantic knowledge about the objects, would predict unimpaired object decision in SD.

This view regarding the independence of object recognition and semantic knowledge is, however, not the only plausible theory, nor does the weight of the available evidence seem to support it. Rogers, Lambon Ralph, Hodges, and Patterson $(2003,2004 b)$ have argued that object recognition arises not from access to dedicated pre-semantic representations but rather from an interaction of perceptual and semantic processing. They have furthermore demonstrated that, when the non-real objects in an object decision task are as typical of their domain as the real objects, SD patients are usually impaired at object decision. The degree of impairment is determined not only by object typicality but also by object familiarity and degree of the patient's semantic deficit.

Additional evidence on the relationship between object recognition and semantic knowledge seems desirable, especially in the form of results from tasks other than object decision. As people go about their daily activities, they are rarely confronted with the need to judge whether something is 'real'. Classification of object tokens as object types, on the other hand, comes closer to something that we do in real life, at least implicitly. We are constantly encountering unfamiliar tokens of familiar types-i.e. specific dogs or bicycles or kettles that we have never seen before. Having learned that a particular response is appropriate to tokens $\mathrm{A}_{1}, \mathrm{~A}_{2}, \ldots$, $A_{m}$ of object-type $A$, it is useful to be able to generalise that response to token $\mathrm{A}_{n}$ when it comes along. There is already some limited evidence from studies of object use to suggest that this kind of generalisation is impaired in SD. Three SD patients (one reported in Snowden, Griffiths, \& Neary, 1994; two in Bozeat, Lambon Ralph, Patterson, \& Hodges, 2002) achieved significantly greater success when asked to demonstrate the use of their own familiar kettles or cameras than when they were given alternative, equally 'good' versions supplied by the experimenter.
In the current study, we adopted the relatively simple goal of determining whether SD patients, who are quite likely to fail in naming or defining familiar objects like a kettle or a mushroom, are also impaired at judging that two different pictures of kettles or mushrooms represent 'the same thing'. The task was structured to determine what sorts of changes in an object - its size, angle of view, colour, exemplar - are likely to make it a 'different thing' for an SD patient when it is still the 'same thing' for a normal participant. By including some SD cases with predominantly left-temporal atrophy and others with equal or even greater abnormality on the right, we also hoped to acquire some evidence regarding the contributions of left- and right-sided structures to this kind of object recognition.

\section{Methods}

\subsection{Participants}

The 10 patient participants (recruited from clinics in either Cambridge or Bath) all fulfilled international consensus criteria for semantic dementia (Neary et al., 1998): anomia, impairment in single word comprehension and impoverished semantic knowledge, with relative preservation of phonology, syntax, visuo-spatial abilities, and day-to-day memory (see Table 1). In all cases, structural brain imaging by MRI $(N=9)$ or CT $(N=1)$ showed the focal atrophy characteristic of SD, involving the polar and inferior regions of the anterior temporal lobe, always bilateral but often highly asymmetrical. All scans were reviewed by two experienced behavioural neurologists (blinded to the experimental data) who classified the scans as involving anterior temporal atrophy that was either predominantly left $(N=5$ : AT, MA, EK, JCh, MK), predominantly right $(N=3$ : $\mathrm{CS}, \mathrm{GT}, \mathrm{PD})$ or about equal on the two sides $(N=2$ : $\mathrm{KH}, \mathrm{JH})$. Illustrative left-predominant, right-predominant and bilateral MR images are displayed in Fig. 1. With reference to evidence concerning, the role of right-hemisphere structures in object recognition, the most important distinction seemed to be between cases with relative sparing of the right temporal lobe versus those with substantial right-temporal involvement. The patients with predominantly right and with bilateral atrophy were therefore merged, thus yielding two patient groups ( $\mathrm{L}>\mathrm{R}$ and $\mathrm{R} \geq \mathrm{L}$ ) with $N=5$ in each. Ten normal participants from the MRC Cognition and Brain Sciences Unit's subject panel, matched to the patients for age and years of education, served as controls.

\subsection{General neuropsychology}

Patients were assessed with the following neuropsychological test battery: the mini mental state examination (MMSE; Folstein, Folstein, \& McHugh, 1975); Ravens coloured progressive matrices (Raven, 1962); the digit span subtest of the Wechsler memory scale-revised (WMS-R; 


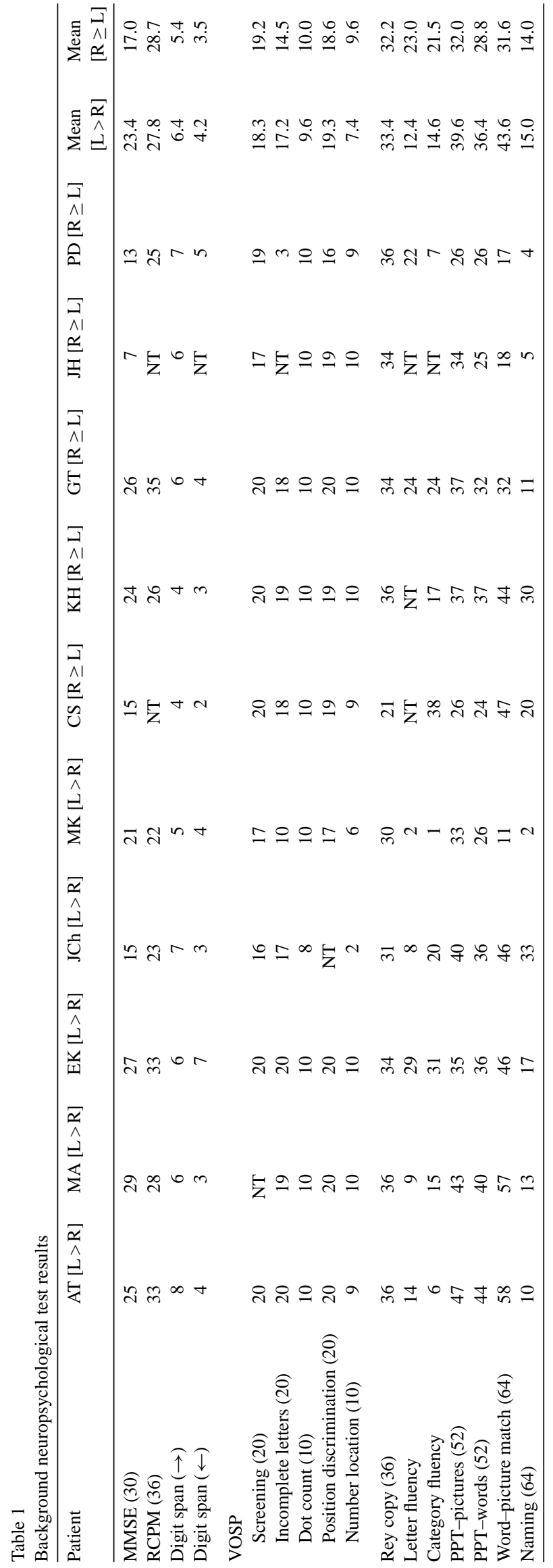

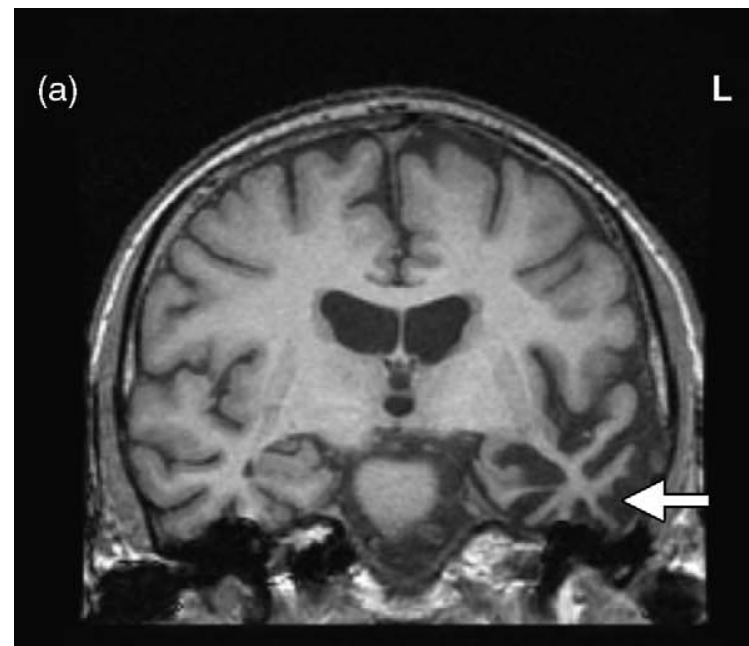

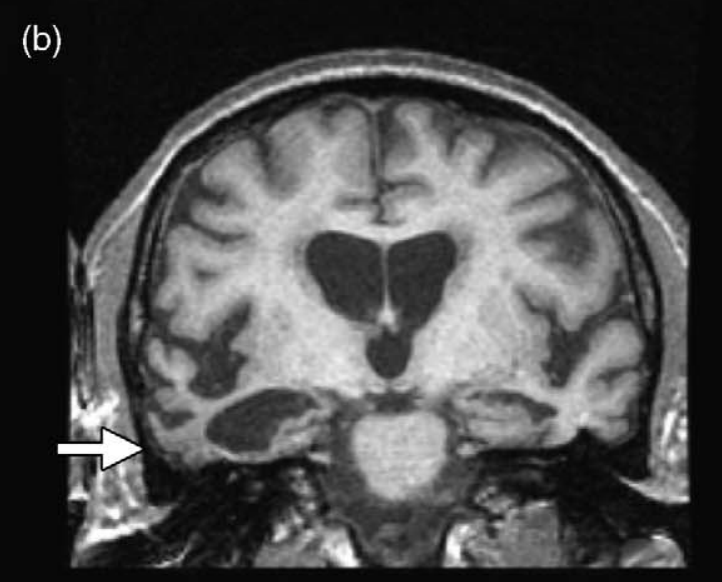

(c)

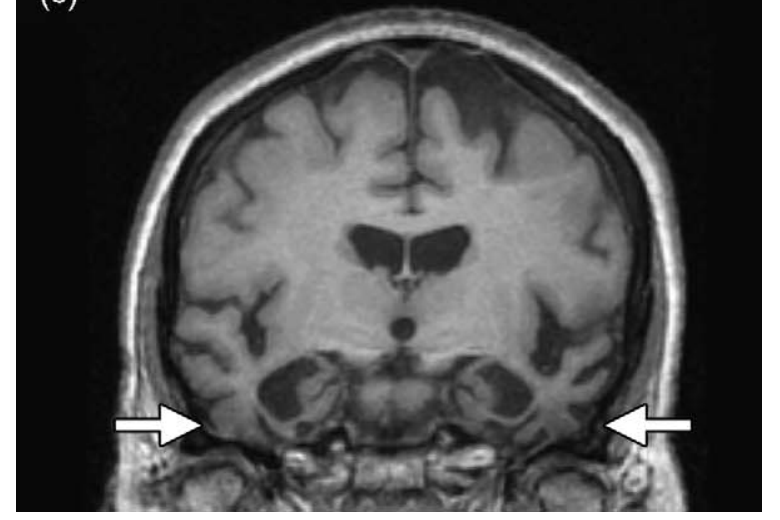

Fig. 1. Coronal views of T-1 MRI scans, at the level of the mid-temporal lobe, for typical cases with: (a) more left-than-right atrophy, (b) more rightthan-left atrophy, and (c) extensive bilateral atrophy.

Wechsler, 1987); subtests of the visual object and space perception battery (VOSP; Warrington \& James, 1991); immediate copy of the Rey complex figure (Rey, 1941); verbal fluency for the letters F, A, and S; category fluency for six different categories; the pyramids and palm trees test of semantic association in both picture and word conditions (Howard \& 
Patterson, 1992); and two tests from the Cambridge semantic battery: (a) word-picture matching in which a single spoken object name $(N=64)$ is to be matched to its corresponding line drawing from a picture array containing the target plus nine within-category foils; (b) naming of the same 64 target pictures.

The results of this neuropsychological testing are displayed in Table 1, where the patients are arrayed in the following order: first the $N=5$ cases with $\mathrm{L}>\mathrm{R}$ atrophy, ranked from least to most impaired on the word-picture matching test of comprehension, and then the $N=5$ cases with $\mathrm{R} \geq \mathrm{L}$ atrophy ranked in the same fashion. As the table indicates, despite the inevitable variability and occasional idiosyncratic scores to be expected in any patient sample, the patients generally displayed the characteristic patterns of SD, with relatively preserved non-verbal problem solving, short-term memory and visuo-spatial function but abnormal performance on all the other assessments, and marked anomia.

Because part of the focus of this study was a comparison of the impact of $\mathrm{L}>\mathrm{R}$ to $\mathrm{R} \geq \mathrm{L}$ atrophy in $\mathrm{SD}$, Table 1 includes mean scores for each of these two subgroups. Although it would of course be desirable to have subgroups matched on MMSE, PPT and/or word-picture matching (i.e. measures of general cognitive and semantic status), this is generally not a feasible goal given limited numbers of SD patients available for testing at any one time. The pattern in Table 1 , in which the $\mathrm{R} \geq \mathrm{L}$ subgroup was distinctly more impaired than the $\mathrm{L}>\mathrm{R}$ cases on these measures, reflects our own and other researchers' experience that $\mathrm{R} \geq \mathrm{L}$ cases often come to neurological attention at a later disease stage and thus tend to be more severe than $L>R$. Note that - despite the greater comprehension deficit in the $\mathrm{R} \geq \mathrm{L}$ subgroup - there was rough equivalence of naming scores in the two subgroups and more prolific output for $R \geq L$ than $L>R$ in both letter and category fluency. This pattern is consistent with our previous observations and modelling of the relationship between comprehension and concept-name production as a function of $\mathrm{L} / \mathrm{R}$ balance of temporal atrophy in SD (Lambon Ralph, McClelland, Patterson, Galton, \& Hodges, 2001).

\subsection{Experiment on object recognition: materials, design and procedure}

The stimuli, consisting of good colour photographs of real objects, taken by digital camera or scanned from photo books, comprised 48 pictures of familiar objects, including animals, fruits/vegetables, household objects, vehicles, etc. Each target object was presented for study initially and then re-presented for recognition but never with precisely the same photograph. In the four different experimental conditions, the target photo for recognition differed from the studied version in, respectively, (i) size, (ii) viewpoint/orientation, (iii) colour, (iv) exemplar (see Fig. 2 for examples). Each condition had its own unique set of objects, with the goal of minimising interference between trials.

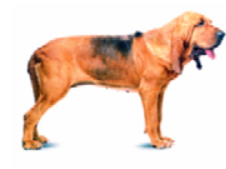

(a)
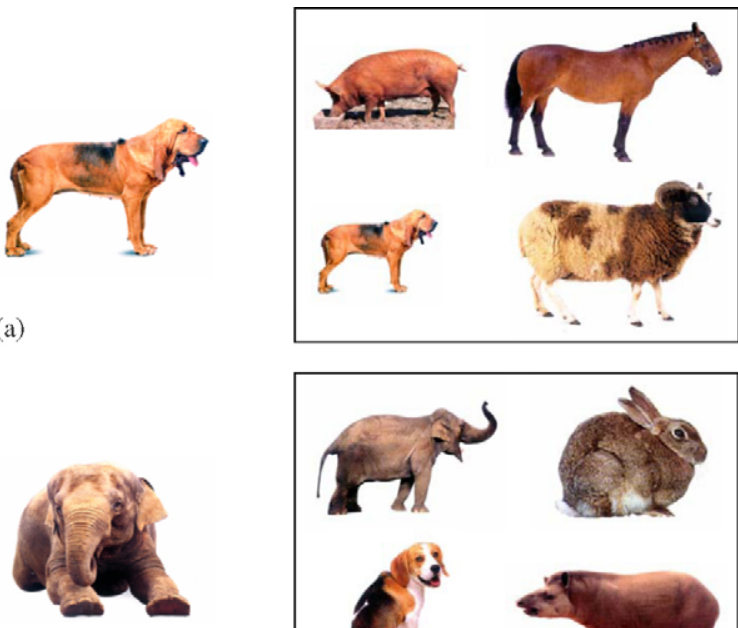

(b)
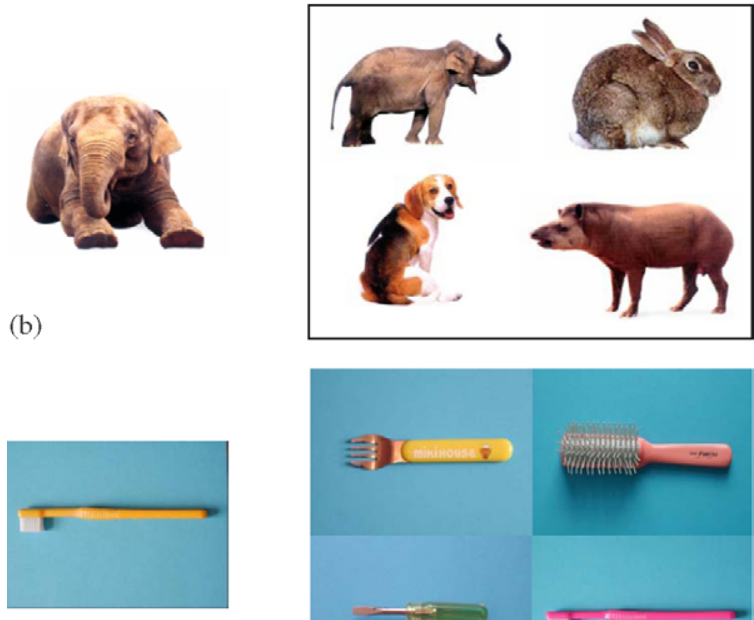

(c)
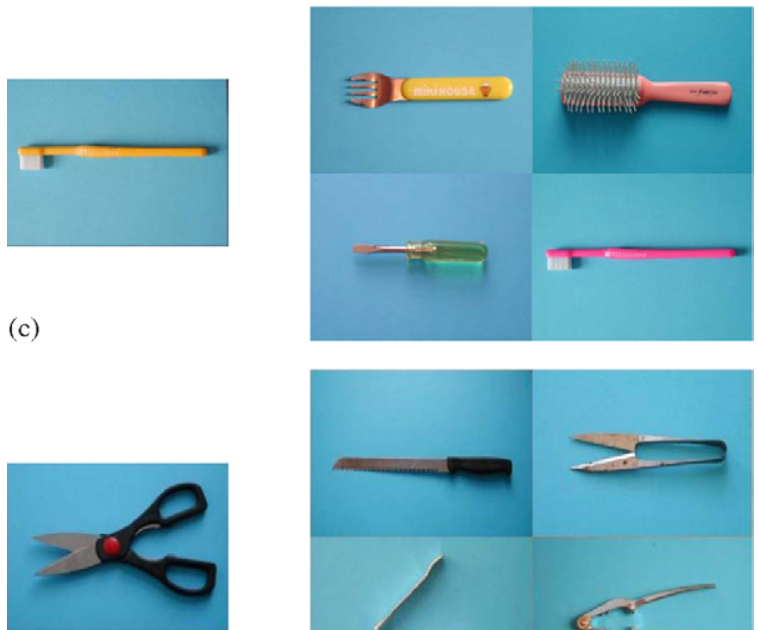

(d)

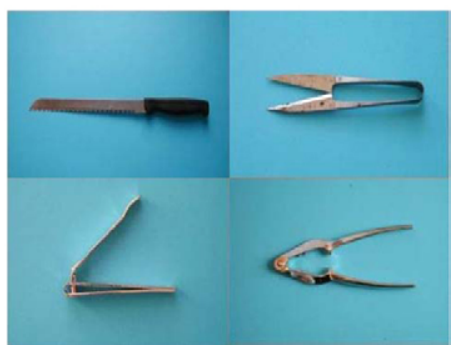

Fig. 2. Example of target objects (on the left) and their corresponding recognition arrays (on the right) from each of the four experimental conditions: (a) change in size, (b) change in view, (c) change in colour, and (d) change in exemplar.

For the size manipulation, the two target photos for any one object (i.e. the photos used for study versus recognition) were precisely identical except that one was a scaled down version of the other. For the viewpoint and colour manipulations, the two target photos for all of the manmade objects (such as cars, toothbrushes, etc.) and some of the natural kinds were identical in all respects except for the viewpoint from which they were photographed or the colour of the object respectively; but this complete identity was not achieved for every natural-kind target in these two conditions. The elephant, for example, was represented by two photographs from animal books showing an elephant standing and an elephant lying down. They are not the same individual but they are typical and largely indistinguishable elephants. Likewise, for the colour-changed apple, we photographed real red and green apples of the same size and shape; side-by-side inspection 
would reveal a difference in the shape of the stem but again, they are to all intents and purposes the same. Finally, for the exemplar manipulation, the two target objects were of course different objects. In the recognition phase of each trial, the target was presented with three foils, chosen to be visually similar to, and/or from the same category as, the target, as illustrated in Fig. 2.

In addition to the four different types of alteration, the experimental design for the patients consisted of two test conditions, immediate and delayed. Each target item was included in both conditions, but without the undesirable consequence of stimulus repetition within a session, by dividing the experiment across two sessions separated by a week or two. In the immediate condition, the patient was first shown a target photo and asked to try to name it. Then this single photo was removed and, without any intervening delay, was replaced by a photo array containing the target response and the three foils. The patient was asked "which one is the same thing, or the same kind of thing, as the picture you were just looking at?" The delayed condition was identical except that, when the initial target photo was withdrawn, the subject engaged in a filler task for 2 min before being shown the test array. In one test session (consisting of half of the target items presented in the immediate condition and the other half in the delayed condition), the filler task for the delayed condition was reading aloud of a series of single words. In the other test session, for which each target object shifted from immediate to delayed testing or vice versa, the filler task was simple arithmetical calculation (written addition, subtraction, multiplication and division problems). Note that neither filler task involves any presentation of pictures or focus on object concepts. Pilot testing established that normal individuals made no errors in the immediate condition; controls were therefore tested only in the delayed condition for the full experiment.

The position of the target amongst the foils was balanced across arrays for each of the four positions on the page. Prior to testing, a set of four practice items was administered to ensure that the task requirements were understood; any patient who seemed to be slow in grasping the idea was allowed to repeat these practice items as many times as necessary. In order to avoid confusion on the patients' part, the block of items for immediate testing was administered first on both test sessions, followed by the delayed block. A further set of practice items was administered to the patients at the beginning of the second block so that they could get used to the delay procedure and the intervening task. Within each immediate or delayed block, items from the four typeof-change conditions were intermixed in random order.

\subsection{Statistics}

Owing to non-homogeneity of variance resulting largely from ceiling effects in the control group, non-parametric Mann-Whitney $U$-tests were used to evaluate the significance of differences in the four experimental conditions between $\mathrm{SD}$ and control groups and between the two SD sub-groups $(\mathrm{L}>\mathrm{R}$ versus $\mathrm{R} \geq \mathrm{L}$ ).

\section{Results}

The control participants as a group scored $97.7 \%$ correct on the delayed recognition task. Overall success rate by the patients was $77.5 \%$ correct $(82.3 \%$ with no delay between target study and recognition test, $72.5 \%$ with a 2 min delay); these values are substantially above chance $(25 \%)$ but well below perfect. Fig. 3 illustrates the mean performance of the controls and of the patients for each of the four experimental conditions. In the size condition, the patients had nearly perfect performance on immediate trials and largely good success even in the delayed condition, resulting in only a trend towards impairment for delayed size judgements by patients versus controls $(Z=2.49, p=0.06)$. The patients as a group had significantly poorer delayed performance than controls in all three of the other conditions, with $\mathrm{Z}$ values ranging from 2.8 to 3.8 , all $p$ values $<0.01$.

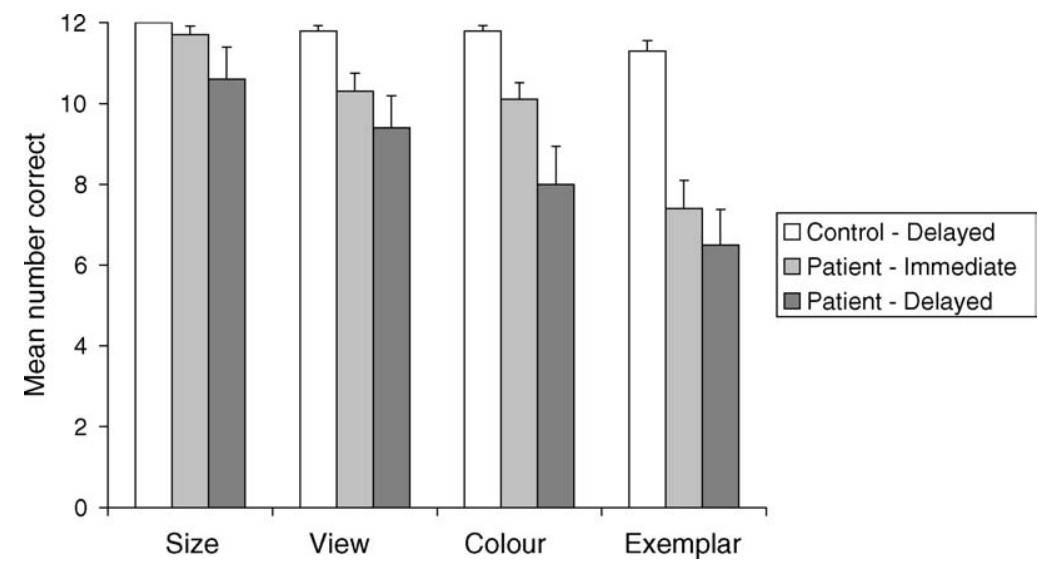

Fig. 3. Mean number of items correct [out of 12] in each of the four experimental conditions in performance by the control group (delayed only) and the SD group (both immediate and delayed tests). Variance bars indicate the standard error of the mean. 


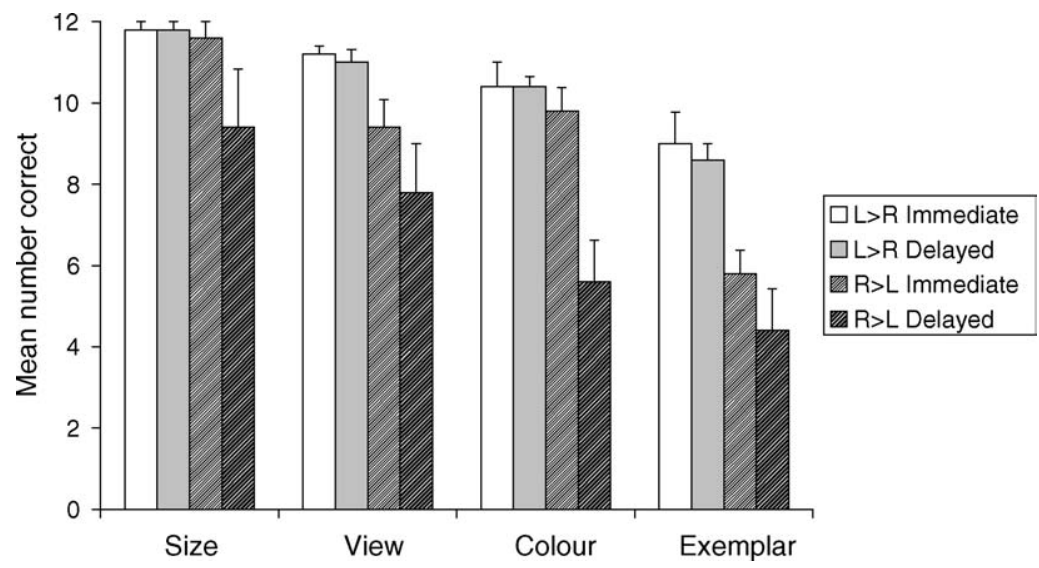

Fig. 4. Mean number of items correct in each of the four experimental conditions, for immediate and delayed tests, separately for the $L>R$ and $R \geq L$ subgroups of SD patients. Variance bars indicate the S.E. of the mean.

Fig. 4 displays the results for SD patients only, separately for immediate versus delayed and also for $\mathrm{L}>\mathrm{R}$ and $\mathrm{R} \geq \mathrm{L}$ subgroups. The scores of the $\mathrm{L}>\mathrm{R}$ sub-group (delayed) were significantly lower than controls (delayed) for the colour $(Z=3.10, p=0.003)$ and exemplar $(Z=3.03, p=0.001)$ con- ditions, marginally lower for viewpoint $(Z=2.16, p=0.075)$ and not different for size $(Z=1.41, p=0.59)$. The scores of the $\mathrm{R} \geq \mathrm{L}$ patients (delayed) were reliably lower than control scores as a function of all four experimental manipulations (size: $Z=3.15, p=0.01$; viewpoint: $Z=2.64, p=0.019$;
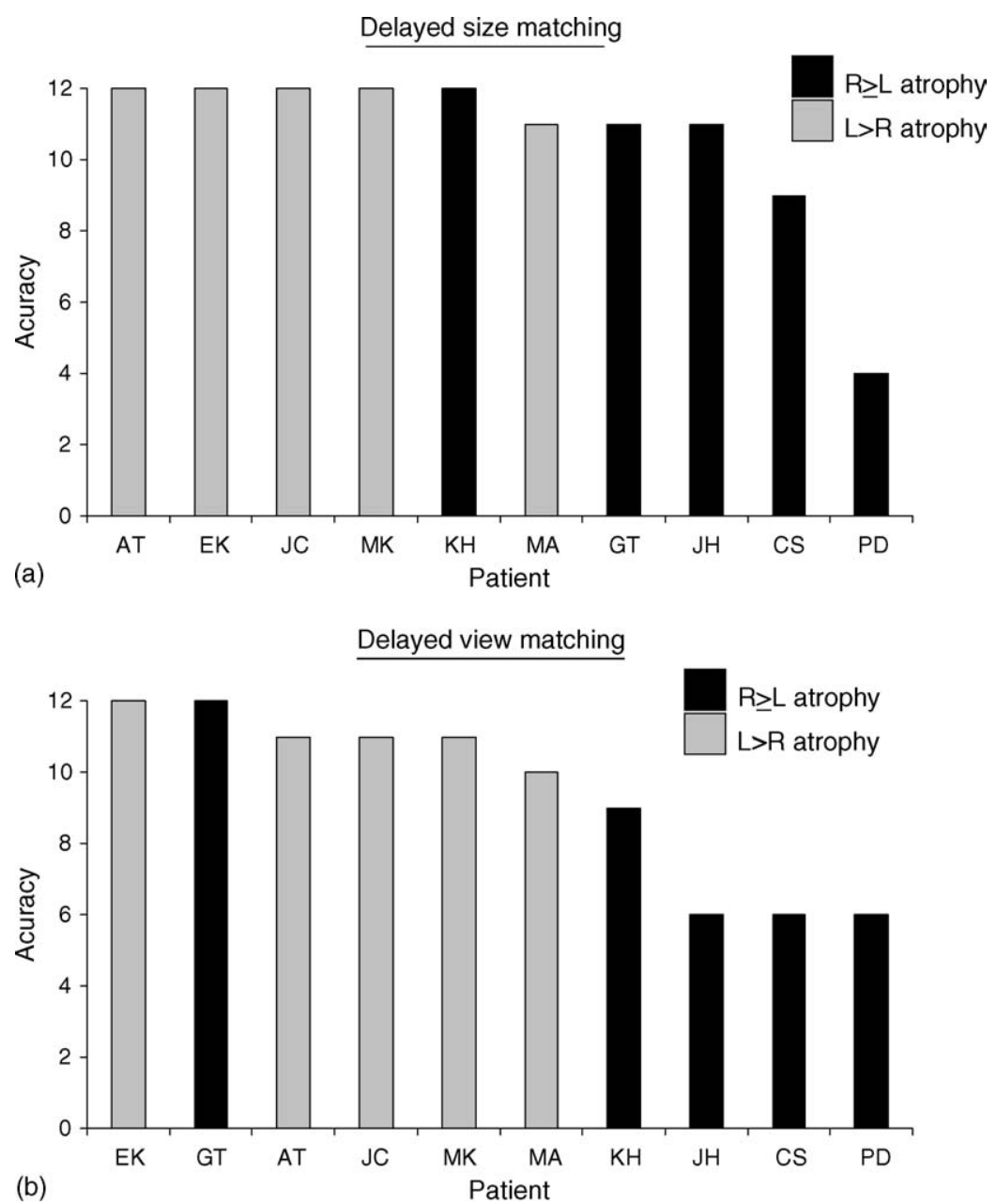

Fig. 5. Accuracy (mean number of items correct out of 12) for individual SD cases in each of the four experimental conditions (delayed only). 

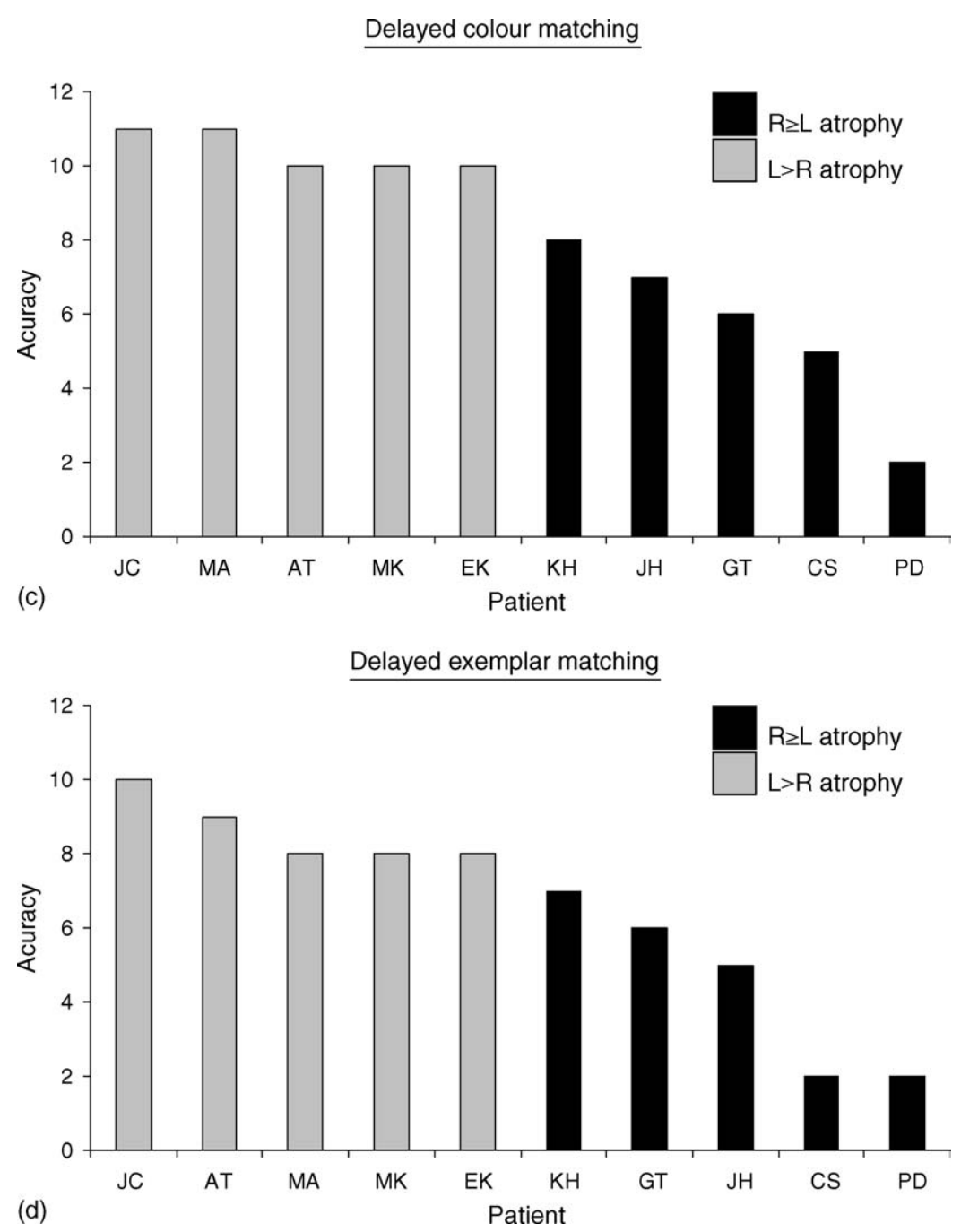

Fig. 5. (Continued).

colour: $Z=3.33, p=0.001$; exemplar: $Z=3.14, p=0.001$ ). Comparison of $\mathrm{L}>\mathrm{R}$ and $\mathrm{R} \geq \mathrm{L}$ revealed significantly poorer performance by the latter subgroup for colour and exemplar changes (in both cases, $Z=2.65, p=0.008$ ), and a borderline disadvantage for $\mathrm{R} \geq \mathrm{L}$ in the size $(Z=1.92, p=0.055)$ and viewpoint $(Z=1.72, p=0.086)$ conditions. In addition to the overall subgroup difference, Fig. 4 reveals that, whereas the delay had no effect on the $L>R$ group, the insertion of this relatively brief interval between study and test resulted in worse performance for the $\mathrm{R} \geq \mathrm{L}$ cases.

Fig. 5a-d displays individual patient scores in each of the four delayed conditions. The only two exceptions to the pattern of poorer performance by $\mathrm{R} \geq \mathrm{L}$ than $\mathrm{L}>\mathrm{R}$ are one 'near displacement' for the size condition (patient KH scoring slightly better than one L > R case, MA) and one 'far displacement' for the view condition (GT scoring slightly better than four $\mathrm{L}>\mathrm{R}$ cases). Otherwise, the pattern holds.

To evaluate this pattern in light of the confounding difference in severity of semantic impairment between the two subgroups, we computed partial correlations of performance in each of the four delayed conditions, partialling out scores on the word-picture matching test, a measure of semantic status on which there was a substantial disadvantage for the $\mathrm{R} \geq \mathrm{L}$ relative to the $\mathrm{L}>\mathrm{R}$ cases. The partial correlation between delayed object recognition and subgroup membership is still reliable for the colour condition $(r=-.82, p=0.007)$ and the exemplar condition $(r=-.77, p=0.016)$ and borderline for the view condition $(r=-.63, p=0.069)$, suggesting that semantic severity does not fully explain the greater deficit on our task for $\mathrm{R} \geq \mathrm{L}$ cases. A visuo-spatial deficit per se is also unlikely to underlie this effect: of the four patients who performed poorly on one of the visual-spatial tests reported in Table 1 (JCh and MK on number location, CS on Rey figure copy, PD on incomplete letters), (a) two of these were in each atrophy subgroup, and (b) no patient showed notably sub-normal performance on more than one of these assessments.

With respect to the nature, rather than just the number, of the patients' incorrect choices: these were of course determined in part by the experimenters' selection of distractor or 
Table 2

Concordance between success in naming the target objects and in recognising them, for the SD patients as a group, combined over immediate and delayed tests and over the four experimental conditions

\begin{tabular}{lclc}
\hline & Naming + & Naming - & Total \\
\hline Recognition + & 268 & 476 & 744 \\
Recognition - & 32 & 184 & 216 \\
Total & 300 & 660 & 960 \\
\hline
\end{tabular}

foil items in the various conditions, and the majority of errors did indeed reflect the manipulated dimensions (e.g. selecting the wrong object with the right colour).

Finally, what about the relationship between the patients' performance on the recognition task and their naming of the target photos? As expected, naming was poor: out of 48 targets presented in each of the two sessions, the patients as a group correctly named an average of $15.6=32.5 \%$ (range, $12.5-58.3 \%$ ) on session 1 and an average of $15.2=31.7 \%$ (range, 10.4-54.2\%) on session 2. Of more interest, however, there was a strikingly high correspondence between success in naming and correct recognition choices. The twoby-two table giving numbers of items correct on both tasks, correct on neither, or correct on one but not the other, is displayed in Table 2, and these values yield $X^{2}$ ( 1 d.f. $)=35.04$, $p<0.0001$. Of the two 'incongruent' cells (that is, correct on one task but not the other), it is not surprising that there are so many entries in the cell for correct recognition without correct naming, since naming is a much harder task than four-alternative forced choice recognition. In the important cell of correct naming followed by an incorrect recognition choice, which we would expect to be virtually empty, there are indeed relatively few entries. Furthermore, more than half of these cases are accounted for by two target objects: dog and horse. These (along with cat) are the most familiar animals in our culture, and it is well known that SD patients over-generalise their names to many other animals (Hodges, Graham, \& Patterson, 1995). 'Correct' naming of a real dog or horse might therefore be spuriously correct; and if these responses are removed, the cell for correct naming followed by incorrect recognition would contain a mere 17 entries out of $960=1.9 \%$ of trials.

\section{Discussion}

This experiment has demonstrated that SD patients have impaired object recognition as measured by the ability to classify two visually different tokens of an object type as the same thing. The impairment was greater for cases whose anterior temporal lobe atrophy included a significant degree of right-sided abnormality than for those with asymmetrical $\mathrm{L}>\mathrm{R}$ atrophy; the $\mathrm{R} \geq \mathrm{L}$ sub-group was also more affected by the imposition of a brief delay between study and test.

The deviation of the patients' scores away from controls in the four conditions followed the coherent order of size < viewpoint $<$ colour $<$ exemplar. Two factors probably contributed to this ordering: (a) whether the relevant alteration ever does, in the real world, signal a change in object type and (b) whether the distractor resembling the original target on the relevant dimension is likely to be an effective lure. Consider a change in size: two photographs that differ in size but are otherwise identical in shape, orientation, colour, proportions of the various parts, etc., never represent different object types; given that the size of a photographed object provides almost no information about its identity, the presence in the response array of a different object of similar size to the original target is probably not an effective lure; therefore this condition should be relatively easy on both criteria. A change in orientation/viewpoint should not signal a different object type, although if critical features of the object are revealed in one viewpoint but obscured in another, the two photos may not be readily identified as the same type of thing. On the other hand, this condition should not lead to many errors because of the second factor: how likely it is that a distractor item will provide a good lure. A change in an object's colour does sometimes signal an object-type change (e.g. lemon and limes can differ little in anything but colour), but this is not so typical. Here, however, the second factor makes the task more difficult: if one has just looked at a picture of a toothbrush with a bright yellow handle, in a subsequent array where the toothbrush handle is pink but there is a spoon with a plastic handle of the same bright yellow as the original target, it is unsurprising that the latter might draw one's attention. The kinds of alterations associated with a change of exemplar, in which almost everything (shape, colour, proportions, etc.) can differ between the two exemplars, do often reflect changes in object type in the real world and it was thus predictable that this condition would be the most difficult for patients with deteriorating conceptual knowledge about objects.

Several previous studies by our research group that were designed to assess the impact of semantic degradation on episodic recognition memory for pictures of objects (Graham, Simons, Pratt, Patterson, \& Hodges, 2000) or famous faces (Simons, Graham, Galton, Patterson, \& Hodges, 2001) included a manipulation similar to that employed here. SD patients achieved essentially normal episodic recognition memory for photographs of objects and faces that were perceptually identical (PI) to the previously studied targets; but when the stimuli had been selected as objects (or people) for which the individual patient's conceptual knowledge was significantly degraded, the patients had significantly impaired memory when shown objects or faces that were perceptually different (PD) from the studied exemplars, e.g. in viewpoint. These experiments were designed to assess recognition memory, and accordingly were composed of a study phase (a whole sequence of target pictures) followed by a 15 min gap (filled with some other task), followed by a recognition memory test. The important conclusion of both of these studies was that new learning could be supported by perceptual as well as semantic information, because 
SD patients performed well at recognition memory, even for items about which they had little semantic information, provided that the pictures at study and test were identical. The results of the PD conditions, which were not critical to this main conclusion, were nevertheless provocative in raising the question of when semantically impaired patients know that two different tokens belong to the same type. This question could not be directly addressed in the previous studies because of the episodic-memory nature of the experimental designs; but it was precisely the focus of the current experiment.

The present data suggest that the ability to identify different tokens as the same type is vulnerable to semantic impairment, even when the task has only a minimal memory component. If two different pictures of a horse or a toothbrush are both meant to activate the same entry in a (posterior cortical) structural description system; and if such activation were thought to be: (a) independent of semantic knowledge about the object in question, and (b) sufficient for the judgement that these are two examples of the same object, then SD patients should have relatively preserved performance even in this task; but they do not. In English, "a horse of a different colour" is a metaphor meaning "that is an entirely different type of thing". This seems to us an apt description for the phenomenon documented here, in which - for semantically impaired patients - changing the colour of a horse or a toothbrush can literally turn it into a different thing.

Our interpretation of these results, in line with that of previously reported results from our group on tasks like object decision and delayed copy-drawing in SD (Rogers et al., 2003, 2004a,b), is this: responses in all of these situations are based not on access to an entry in a structural description system but rather on interactive processing between perceptual and conceptual information. In fact, our interpretation is intended to challenge the assumptions listed above under which one might have predicted SD performance to be unimpaired on this recognition task: i.e. that classifying different tokens as the same type is achieved by access to a structural description entry that is independent of semantic knowledge. Our claim instead is that, when conceptual knowledge about the objects in question becomes degraded, then it is increasingly difficult for the person to recognise an object as belonging to the same class as another instance, or (in the object decision paradigm) as a real rather than a plausible but non-real thing, or (in the delayed copy paradigm) as a bird rather than a generic animal and hence deserving of two legs rather than four. The direct relevance of semantic knowledge to performance in these tasks has been demonstrated in various ways, for example in the delayed copy task by a dramatic advantage for familiar objects preselected as still relatively 'known' versus now 'degraded' for the specific patient (Jefferies, Jones, Bateman, \& Lambon Ralph, 2005). In the present study, it would have been almost impossibly difficult to create different sets of 'known' versus 'degraded' photographs for each condition for each of
10 patients. There is however support for the impact of semantic knowledge on this object recognition task in the high concordance between the patients' success in naming the objects and in recognising different tokens as the same type.

What of the consistently greater impairment on this task in the $\mathrm{R} \geq \mathrm{L}$ than the $\mathrm{L}>\mathrm{R}$ subgroup? Although it is not possible to rule out the relevance of the somewhat greater semantic deficit in these particular $\mathrm{R} \geq \mathrm{L}$ cases, we also hypothesise a genuine difference in the contributions of left and right temporal lobes to the processes required for object recognition. First, as noted in the Section 1, there is considerable evidence to suggest a somewhat specialised role of posterior right-hemisphere regions in perceptual processing of the visual structure of objects. Second, we propose that object recognition requires communication and interaction between the posterior structures essential for perceptual analysis and the anterior temporal regions critical for semantic processing. Although, by our hypothesis, semantic knowledge relies on the anterior temporal lobes bilaterally, it is plausible that the right temporal pole has a somewhat more influential role in semantic processing of visually presented objects because the perceptual information that interacts with semantic representations comes more strongly from right than left posterior regions. This would parallel our account of the observation that object naming is more impaired in $\mathrm{L}>\mathrm{R}$ than $\mathrm{R}>\mathrm{L}$ SD patients: on the basis of behavioural studies and a connectionist model, Lambon Ralph et al. (2001) argued that, because the phonological representations for speech are left lateralised, the left side of the bilateral semantic network may form stronger connections to speech output than the right side of this network does. In the current account, the argument is that $\mathrm{R} \geq \mathrm{L}$ patients will tend to be more impaired in object recognition than their $\mathrm{L}>\mathrm{R}$ counterparts because, despite relatively normal early visual processing in the posterior right hemisphere, this successful processing fails to activate the information required for object recognition in the damaged right anterior temporal cortex. An equivalent abnormality at the left temporal pole will have less impact on object recognition because the right posterior $\leftrightarrow$ left anterior interaction was always somewhat weaker and less critical for visual object recognition.

In conclusion, we propose that $\mathrm{SD}$ is a disorder that results in both over- and under-generalisation relative to normal conceptual processing. Instances of over-generalisation are widely documented, in the patients' naming (one of the previous SD patients in our cohort, looking at a picture of a zebra, said "it's a horse, isn't it?" Then, pointing to the stripes, she said, "but what are these funny things for?"). This phenomenon is also apparent on tests of lexical and object decision (Patterson et al., in press; Rogers et al., 2004b). The conceptual deterioration characteristic of SD also, however, makes the specific features of an object more salient, such that two representations of an object that are equivalent for normal individuals are now "horses of different colours" for the patients. 


\section{Acknowledgements}

The research reported here was supported in part by an MRC Programme Grant to author JRH and by an NIMH Center Grant to authors KP and MALR. We are grateful to Dr. Rhys Davies and Dr. Anna Woollams for help with the MRI pictures and other illustrations for this paper.

\section{References}

Bozeat, S., Lambon Ralph, M. A., Graham, K. S., Patterson, K., Wilkin, H., Rowland, J., Rogers, T. T., \& Hodges, J. R. (2003). A duck with four legs: Investigating the structure of conceptual knowledge using picture drawing in semantic dementia. Cognitive Neuropsychology, 20, $27-47$.

Bozeat, S., Lambon Ralph, M. A., Patterson, K., Garrard, P., \& Hodges, J. R. (2000). Non-verbal semantic impairment in semantic dementia. Neuropsychologia, 38, 1207-1215.

Bozeat, S., Lambon Ralph, M. A., Patterson, K., \& Hodges, J. R. (2002). The influence of personal familiarity and context on object use in semantic dementia. Neurocase, 8, 127.

Coltheart, M. (2004). Are there lexicons? Quarterly Journal of Experimental Psychology A, 57, 1153-1171.

Folstein, M. F., Folstein, S. E., \& McHugh, P. R. (1975). Mini-mental state. A practical method for grading the cognitive state of patients for the clinician. Journal of Psychiatric Research, 12, 189-198.

Galton, C. J., Patterson, K., Graham, K., Lambon Ralph, M. A., Williams, G., Antoun, N., Sahakian, B., \& Hodges, J. R. (2001). Differing patterns of temporal atrophy in Alzheimer's disease and semantic dementia. Neurology, 57, 216-225.

Gerlach, C., Law, I., Gade, A., \& Paulson, O. B. (1999). Perceptual differentiation and category effects in normal object recognition: A PET study. Brain, 122, 2159-2170.

Graham, K. S., Simons, J. S., Pratt, K. H., Patterson, K., \& Hodges, J. R. (2000). Insights from semantic dementia on the relationship between episodic and semantic memory. Neuropsychologia, 38, 313-324.

Hodges, J. R., Graham, N. L., \& Patterson, K. (1995). Charting the progression in semantic dementia: Implications for the organisation of semantic memory. Memory, 3, 463-495.

Hodges, J. R., Patterson, K., Oxbury, S., \& Funnell, E. (1992). Semantic dementia: Progressive fluent aphasia with temporal lobe atrophy. Brain, 115, 1783-1806.

Howard, D., \& Patterson, K. (1992). The Pyramids and Palm Trees Test: A Test of Semantic Access from Words and Pictures. Bury St. Edmunds, UK: Thames Valley Test Company.

Humphreys, G. W., Riddoch, M. J., \& Quinlan, P. T. (1988). Cascade processes in picture identification. Cognitive Neuropsychology, 5, 67-103.

Imura, T. (1943). Aphasia: Characteristic symptoms in Japanese. Psychiatra et Neurologia Japonica, 46, 196-218.

Jefferies, E., Jones, R. W., Bateman, D., \& Lambon Ralph, M. A. (2005). A semantic contribution to nonword recall? Evidence for intact phonological processes in semantic dementia. Cognitive Neuropsychology, 22, 183-212.

Kellenbach, M. L., Brett, M., \& Patterson, K. (2001). Large, colorful, or noisy? Attribute- and modality-specific activations during retrieval of perceptual attribute knowledge. Cognitive and Affective Behavioral Neuroscience, 1, 207-221.

Kellenbach, M. L., Hovius, M., \& Patterson, K. (2005). A PET study of visual and semantic knowledge about objects. Cortex, 41, 107-118.
Lambon Ralph, M. A., \& Howard, D. (2000). Gogi aphasia or semantic dementia? Simulating and assessing poor verbal comprehension in a case of progressive fluent aphasia. Cognitive Neuropsychology, 17, 437-465.

Lambon Ralph, M. A., McClelland, J. L., Patterson, K., Galton, C. J., \& Hodges, J. R. (2001). No right to speak? The relationship between object naming and semantic impairment: Neuropsychological evidence and a computational model. Journal of Cognitive Neuroscience, 13, 341-356.

Lauro-Grotto, R., Piccini, C., \& Shallice, T. (1997). Modality-specific operations in semantic dementia. Cortex, 33, 593-622.

Mummery, C. J., Patterson, K., Price, C. J., Ashburner, J., Frackowiak, R. S. J., \& Hodges, J. R. (2000). A voxel-based morphometry study of semantic dementia: Relationship between temporal lobe atrophy and semantic memory. Annals of Neurology, 47, 36-45.

Neary, D., Snowden, J. S., Gustafson, L., Passant, U., Stuss, D., Black, S., Freedman, M., Kertesz, A., Robert, P. H., Albert, M., Boone, K., Miller, B. L., Cummings, J., \& Benson, D. F. (1998). Frontotemporal lobar degeneration: A consensus on clinical diagnostic criteria. Neurology, 51, 1546-1554.

Patterson, K., Lambon Ralph, M. A., Jefferies, E., Woollams, A., Jones, R., Hodges, J. R., \& Rogers, T.T. (in press). 'Pre-semantic' cognition in semantic dementia: Six deficits in search of an explanation. Journal of Cognitive Neuroscience.

Raven, J. C. (1962). Coloured Progressive Matrices. London: HK Lewis.

Rey, A. (1941). L'examen psychologieque dans les cas d'encephalopathie traumatique. Archives de Psychologie, 28, 286-340.

Rogers, T. T., Lambon Ralph, M. A., Garrard, P., Bozeat, S., McClelland, J. L., Hodges, J. R., \& Patterson, K. (2004). The structure and deterioration of semantic memory: A neuropsychological and computational investigation. Psychological Review, 111, 205-235.

Rogers, T. T., Lambon Ralph, M. A., Hodges, J. R., \& Patterson, K. (2003). Object recognition under semantic impairment: The effects of conceptual regularities on perceptual decisions. Language and Cognitive Processes, 18, 625-662.

Rogers, T. T., Lambon Ralph, M. A., Hodges, J. R., \& Patterson, K. (2004). Natural selection: The impact of semantic impairment on lexical and object decision. Cognitive Neuropsychology, 21, 331352.

Simons, J. S., Graham, K. S., Galton, C. J., Patterson, K., \& Hodges, J. R. (2001). Semantic knowledge and episodic memory for faces in semantic dementia. Neuropsychology, 15, 101-114.

Snowden, J. S., Goulding, P. J., \& Neary, D. (1989). Semantic dementia: A form of circumscribed cerebral atrophy. Behavioural Neurology, 2, $167-182$.

Snowden, J. S., Griffiths, H., \& Neary, D. (1994). Semantic dementia: Autobiographical contribution to preservation of meaning. Cognitive Neuropsychology, 11, 265-288.

Snowden, J. S., \& Neary, D. (2002). Relearning of verbal labels in semantic dementia. Neuropsychologia, 40, 1715-1728.

Tanabe, H., Ikeda, M., Nakagawa, Y., Yamamoto, H., Ikejiri, Y., Kazui, H., Hashikawa, K., \& Harada, K. (1992). Gogi (word meaning) aphasia and semantic memory for words. Higher Brain Function Research, 12, 153-167 [in Japanese with an English abstract].

Warrington, E. K., \& James, M. (1988). Visual apperceptive agnosia: A clinico-anatomical study of three cases. Cortex, 24, 13-32.

Warrington, E. K., \& James, M. (1991). The Visual Object and Space Perception Battery. Bury St. Edmunds, England: Thames Valley Test Company.

Wechsler, D. (1987). Wechsler Memory Scale-Revised. San Antonio: Psychological Corporation. 